\title{
Artigo/Article
}

\section{Ecologia de Anopheles (Nyssorhynchus) darlingi Root em área de implantação de empreendimento hidrelétrico, na divisa dos Estados do Mato Grosso do Sul e São Paulo}

\author{
Ecology of Anopheles (Nyssorhynchus) darlingi Root in the installation area of a hydroelectric \\ scheme on the border between the States of Mato Grosso do Sul and São Paulo
}

\author{
Almério de Castro Gomes ${ }^{1}$, Marcia Bicudo de Paula ${ }^{1}$, Delsio Natal ${ }^{1}$ e Sabina Lea Davidson Gotlieb ${ }^{1}$
}

\begin{abstract}
RESUMO
Introdução: Hidrelétricas alteram o fluxo das águas e provocam impactos sobre a composição de mosquitos, justificando-se essa pesquisa. O objetivo da pesquisa foi estudar anofelinos de área sob a influência de um novo lago e avaliar a vulnerabilidade relativa à malária. Métodos: Foram feitas coletas de Anopheles nas margens da Represa Porto Primavera, durante as fases do alagamento até sua cota máxima. Utilizaram-se as técnicas: atrativa humana, de armadilha de Shannon e concha entomológica. Os indicadores Riqueza e Diversidade foram utilizados para medir o impacto. A análise das distribuições temporais foi realizada pelo teste Mann-Whitney, considerando localidade, cota e método de captura como variáveis independentes $(\alpha=0,05)$. Resultados: A densidade de Anopheles darlingi oscilou entre as localidades A, B e C, sendo que os maiores picos foram para $\mathbf{B}$ e C. Com a estabilidade do lago, no último nível, evidenciou-se a tendência de redução da densidade de Anopheles darlingi. Conclusões: Sugere-se que o risco de autoctonia de malária nas proximidades do lago permanece inalterado, ficando o alerta para esporádicas infecções humanas.
\end{abstract}

Palavras-chaves: Hidrelétrica. Malária. Plasmódios. Sucessão ecológica.

\begin{abstract}
Introduction: Hydroelectric schemes modify the water flow and cause an impact on mosquito composition, thus justifying investigations. The aim of this study was to study anophelines in the area under the influence of a new lake and to evaluate their relative vulnerability to malaria. Methods: Anopheles specimens were collected from the edges of the Porto Primavera reservoir, during the phases of reservoir filling until its maximum level was reached. The techniques used were attraction to humans, Shannon traps and entomological scoops. The richness and diversity indexes were used to measure the impact. The temporal distribution analysis was performed using the Mann-Whitney test, taking the site, level and capture method as independent variables $(\alpha=0.05)$. Results: The density of Anopheles darlingi oscillated among the sites A, B and C, and the biggest peaks were located at $\mathbf{B}$ and $\mathbf{C}$. After the lake reached its stable maximum level, there was a tendency for the density to decrease. Conclusions: It is suggested that the risk of autochthonous malaria in the vicinity of the lake remained unchanged, but it is necessary to remain alert regarding sporadic human infections.
\end{abstract}

Key-words: Hydroelectric. Malaria. Plasmodium. Ecological succession.

1. Departamento de Epidemiologia, Faculdade de Saúde Pública, Universidade de São Paulo, São Paulo, SP. Endereço para Correspondência: Dr. Almérico de Castro Gomes. Dept ${ }^{\circ}$. Epidemiologia/FSP/USP. Av. Dr. Arnaldo 715, 01246-904 São Paulo, SP.

Tel: 5511 3061-7738

e-mail: agcastro@usp.br

Recebido para publicação em 20/10/2009

Aceito em 05/03/2010

\section{INTRODUÇÃO}

A Região Amazônica, detentora de fauna rica de anofelinos, é responsável por $99 \%$ dos casos humanos notificados de malária no país ${ }^{1}$. Fora de seus limites, registram-se casos isolados ou surtos da doença, introduzidos por portadores de plasmódios, geralmente procedentes das áreas de transmissão sustentada ${ }^{2,3}$.

Na maioria das situações a malária está associada às transformações do ambiente silvestre, ocasiões que propiciam a exposição de contingente populacional suscetível, entretanto a continuidade do estado de risco pode ser reduzida sob os efeitos da extensão da devastação ${ }^{4}$. A região Noroeste do Estado de São Paulo é um exemplo da transição da infecção, pois passou de período de transmissão endêmica para a fase de registro esporádico de casos, na medida em que a mata original cedeu seu espaço ao ambiente modificado. Para todo o estado, a malária teve declínio até a década de 1980, quando, então, assumiu o perfil epidemiológico focal e isolado 5 . Os registros de casos autóctones totalizaram 388 no período de 1990 a 2006 e os importados foram de $8.025^{6}$.

As ações empreendidas pela Campanha de Erradicação daMalária e as mudanças socioeconômicas e ambientais pelas quais passou o Estado de São Paulo, certamente, deram suas contribuições para que fosse atingido o quadro epidemiológico atual da doença. A redução histórica da notificação da doença, por minimizar sua importância, refletiu no desinteresse por estudos ecológicos, e, como consequência, a vigilância tornou-se carente de avanços no conhecimento sobre a estrutura populacional dos anofelinos desse estado. Para Johnson e cols ${ }^{7}$, a pesquisa ecológica é importante diante das respostas dos anofelinos às transformações antrópicas, porquanto, esse estudo focaliza a riqueza e a diversidade e, a partir desses parâmetros, procede à análise do potencial de transmissão. 
Diante da hipótese de que os empreendimentos envolvendo o ambiente alteram a paisagem e a composição específica de Anopheles ${ }^{8}$, a represa de Porto Primavera desponta como um novo fator de estímulo à adaptação e expansão dos principais vetores, como Anopheles (Nyssorhynchus) darlingi e An (Nys) albitarsis s.l. aos novos ambientes.

No entorno do lago da hidroelétrica, a chance do retorno da malária se torna provável, pois a região abriga um assentamento com milhares de pessoas instaladas em local alcançado pela dispersão dos anofelinos. A possibilidade da introdução de portadores de plasmódios, bem como a exposição dos indivíduos ao estreito relacionamento com anofelinos torna a área vulnerável. Corrobora com essa assertiva a permanência dos Plasmodium vivaxe Plasmodium falciparum em primata não humano na área do estudo ${ }^{1}$, mostrando que temporariamente esses animais poderiam ter a atribuição de atuarem como fonte de infecção para a população humana local. Alerta-se também, para uma possível urbanização da doença, como

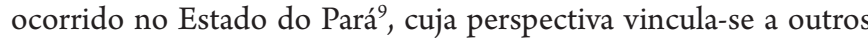
empreendimentos envolvendo cidades do sudeste e sul do país ${ }^{10}$.

É nesse sentido que o conhecimento sobre anofelinos relativos ao estabelecimento de um empreendimento para geração de energia, no Estado de São Paulo, merece uma avaliação. Portanto, definiu-se como objetivo, obter parâmetros relativos à riqueza e à diversidade sob a influência das alterações produzidas pelo estabelecimento do lago artificial e seus reflexos sobre o possível retorno da malária humana na área de estudo.

\section{MÉTODOS}

O trabalho de campo, de desenho prospectivo, para avaliar a riqueza e a diversidade de anofelinos, ocorreu nas margens direita e esquerda do rio Paraná, na divisa entre os Estados do Mato Grosso do Sul e São Paulo ${ }^{1,11}$.

Foram definidas três áreas em função do tipo de solo, com formação de lagoas ou inundações temporárias e vegetação residual, entremeados por ambientes antropizados no município de Bataguassu, Mato Grosso do Sul denominado localidade $\mathbf{A}^{12} \mathrm{e}$ no complexo Lagoa São Paulo no município de Presidente Epitácio, Estado de São Paulo, denominada como localidade B, e localidade C à margem esquerda do Rio do Peixe, tributário do Rio Paraná, distante $10 \mathrm{~km}$ da localidade $\mathbf{B}^{1}$.

A distribuição da população humana estava situada no eixo entre as localidades $\mathbf{B}$ e $\mathbf{C}$, sob a forma de Reassentamento denominado Lagoa São Paulo (RLSP), com mais de mil habitações, agrovilas e lotes de terrenos, geralmente com uma habitação e um centro comercial do Distrito de Campinal. Em toda a área de estudo havia cerca de 4.500 habitantes e uma população flutuante muito variável, que utiliza a represa como área de lazer e pesca.

Para a captura mensal dos mosquitos na mata foram utilizados a Técnica Atrativa Humana por 24 horas (TAH 24h), a Armadilha de Shannon durante 15 horas (AS 15h), das 17 às 8 horas.

Todas as capturas foram realizadas por dois coletadores com equipamentos de proteção pessoal para evitar eventuais picadas de mosquitos.

Os períodos de aplicação da técnica de captura foram, na localidade A, de fevereiro de 1998 a janeiro de 1999 (cota 247m) (nível normal do rio). Na B, de julho de 1997 até fevereiro de 2002 (cotas $247 \mathrm{~m}, 253 \mathrm{~m}, 257 \mathrm{~m}$ ), enquanto na $\mathbf{C}$, de abril de 2001 a fevereiro de 2002 (cota $257 \mathrm{~m}$ ) (cota máxima do reservatório).

Para a pesquisa de formas imaturas foram visitadas todas as coleções líquidas e, com auxílio de uma concha de $500 \mathrm{ml}$ de volume, transferiam as mesmas para potes plásticos que, no laboratório da Faculdade de Saúde Pública da Universidade de São Paulo, se processava a identificação de todos os indivíduos.

Utilizou-se como Índice de Riqueza o número de espécies anofélicas, enquanto a diversidade dos Anopheles foi mensurada pelo Índice de Brillouin ${ }^{6,13}$, nas três localidades, considerando ainda a cota original $(247 \mathrm{~m})$ do rio Paraná e as cotas 253 e $257 \mathrm{~m}$ da barragem. Este índice estima a diversidade das espécies, medindo indivíduos de cada espécie, em cada comunidade e incorpora a abundância relativa dos espécimes capturados diante da série temporal das inundações provocadas pela Hidrelétrica Porto Primavera. Para essas comparações foram realizados testes de Mann-Whitney para índices de riqueza e diversidade em um nível de significância de $\alpha=0,05$, analisando as distribuições de frequências segundo a localidade, cota e método de captura como variáveis independentes. As análises foram executadas por meio do programa computacional SPSS, versão 12.

\section{RESULTADOS}

As capturas de mosquito resultaram em 53.863 indivíduos Culicidae, pertencentes a 11 gêneros, nas três fases de investigação, que corresponderam às etapas da construção à operacionalização da Usina Porto Primavera. Para o gênero Anopheles registrou um total de 12.652 exemplares distribuídos em 14 espécies (Tabela 1).

A localidade A apresentou 1.199 anofelinos coletados, de um total de 3.577 culicídeos, entre imaturos $(113 / 625)$ e alados (1.086/2.952), nos anos de 1998 e 1999. Foram capturadas 11 espécies de Anopheles sem transcorrer novos impactos ambientais na localidade, sendo evidenciadas An triannulatus (16,9\%), An braziliensis (10,5\%), An albitarsis (3,4\%), An darlingi (1,3\%) e An galvaoi (0,6\%). Na localidade B, onde todas as capturas estiveram em torno da Lagoa São Paulo, a fauna anofélica foi de 3.479 (375 imaturos e 3.104 alados) de um total de 31.570 culicídeos. Dentre as 12 espécies de Anopheles encontradas, registrou-se a inclusão de An argyritarsis e An parvus e a exclusão de An oswaldoi e An peryassui, em relação a localidade A. Na localidade $\mathbf{C}$, os culicídeos (18.716 exemplares) foram capturados ao longo da margem do rio do Peixe estendida por uma várzea, que sofria inundações periódicas pelo transbordamento do mesmo, durante as chuvas de verão. Com a formação da represa este fenômeno deixou de existir, pois sua inundação passou a ser permanente. Nove espécies de Anopheles sobressaíram 7.974 exemplares (238 imaturos e 7.736 alados) em relação aos locais A e B (Tabela 1).

Nas capturas das formas imaturas, em 1997 e 1998, houve baixo rendimento nas margens da Lagoa São Paulo e várzea adjacente. Diferentemente, na primeira inundação, o número de imaturos aumentou significativamente, para depois declinar nos anos seguintes, quando o nível da barragem atingiu $257 \mathrm{~m}$. Nesse quadro, chama atenção a raridade do encontro da espécie An darlingi, no complexo Lagoa São Paulo (período 1997/1998). Ao contrário, na cota $253 \mathrm{~m}$ esta espécie, sob a forma imatura, passou a ser tão comum quanto An albitarsis s.l. e An triannulatus. Na cota $257 \mathrm{~m}$, a queda numérica de indivíduos foi acentuada. 


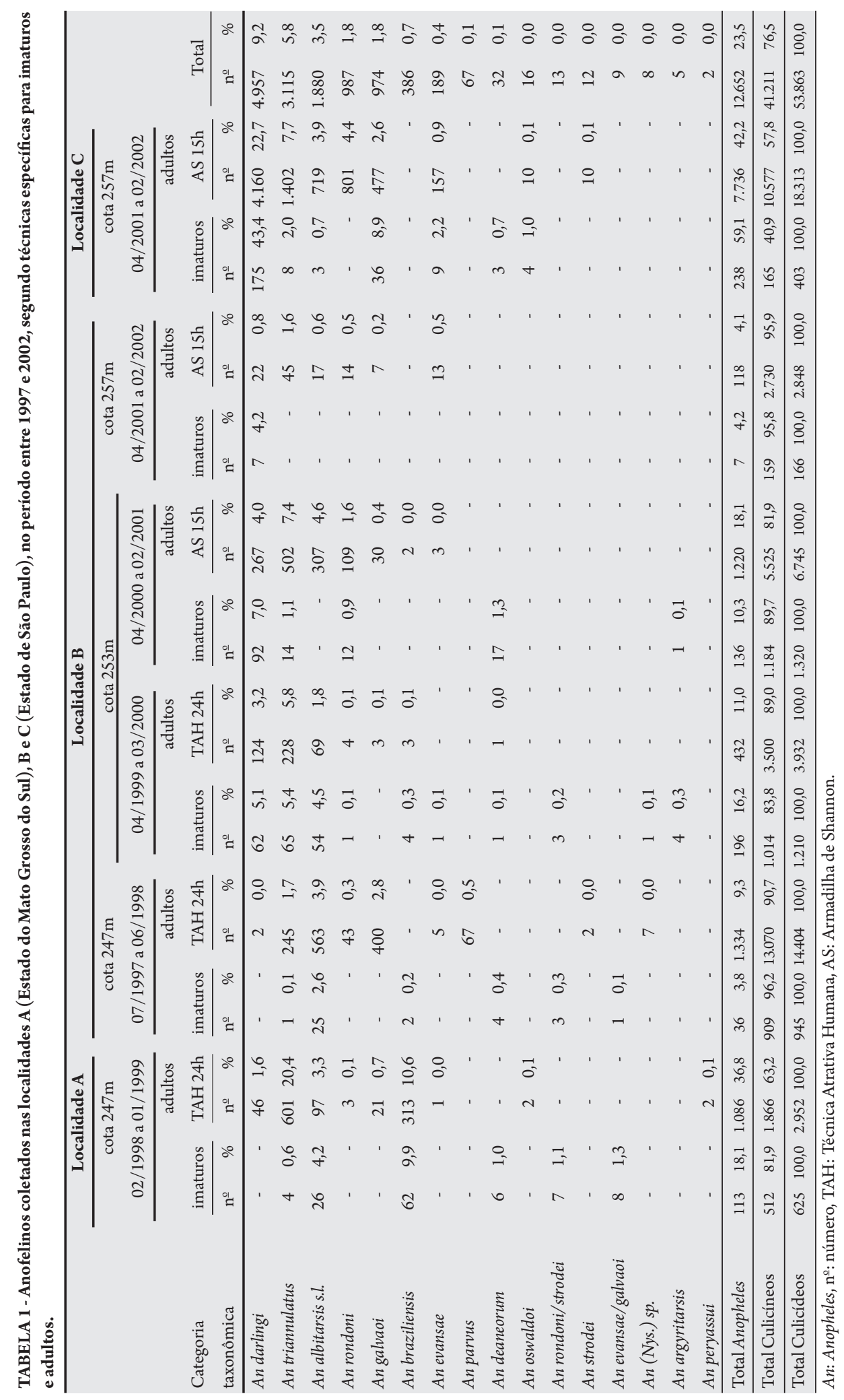

Nas formas adultas, antes da inundação (cota $247 \mathrm{~m}$ ), o resultado para as localidades $\mathbf{A}$ e $\mathbf{B}$ foram diferentes em termos de abundância, com predominância de An triannulatus (20,4\%), An braziliensis (10,6\%) e An albitarsis s.l. (3,3\%), enquanto que, na localidade $\mathbf{B}$ houve uma discreta presença de An albitarsis s.l. (3,9\%) e An galvaoi (2,8\%) e An triannulatus (1,7\%). Ao comparar essas duas localidades, os índices de riqueza e diversidade não apresentaram diferenças significativas estatísticas, tanto para os adultos como para os imaturos. Na localidade
C, An darlingi predominou (22,7\%) seguido de An triannulatus (7,7\%), An rondoni (4,4\%) e An albitarsis s.1 (3,9\%) (Tabela 1). Para os Anopheles adultos, da localidade $\mathbf{B}$ houve diferenças significativas do ponto de vista estatístico, para riqueza $(\mathrm{p}=0,001)$ e diversidade $(\mathrm{p}=0,04)$, ao compará-las nas cotas $247 \mathrm{~m}$ e $253 \mathrm{~m}$ pela TAH $24 \mathrm{~h}$ e $253 \mathrm{~m}$ e $257 \mathrm{~m}$ pela AS $15 \mathrm{~h}(\mathrm{p}=0,01 ; \mathrm{p}=0,04)$. E entre as localidades B e C, na cota $257 \mathrm{~m}$, a riqueza e a diversidade também apresentaram diferenças estatisticamente significantes $(p=0,001 ; p=0,01)$. 
No caso dos adultos de An darlingi, a detecção foi de 1,6\%, para a localidade A, e abaixo de $1 \%$, para $\mathbf{B}$, na cota $247 \mathrm{~m}$ e $3,2 \%$ na cota $253 \mathrm{~m}$, comparados pela mesma técnica de captura (TAH 24h). No entanto, pela a AS 15h, depois da primeira e segunda inundação, na localidade B, An darlingi apresentou-se em $4 \%$ e 0,8\%, respectivamente, e 22,7\% na localidade $\mathbf{C}$, após a segunda inundação. $\mathrm{E}$ a pesquisa de imaturos revelou-se expressiva para An darlingi na localidade B, após a primeira (7\%) e segunda inundação $(4,2 \%)$ e na localidade C, após a segunda inundação $(43,4 \%)$ (Tabela 1$)$.

\section{DISCUSSÃO}

No ambiente estudado, os habitats naturais foram previamente comprometidos, pela ação humana, enquanto a represa acrescentou novas interferências. Por meio da análise de riqueza e diversidade de mosquitos Culicidae, particulariza-se An darlingi e outros vetores secundários de plasmódios, objetivando discutir a hipótese de possível mudança no padrão esporádico de ocorrência de casos de malária humana, nas proximidades do lago artificial.

$\mathrm{Na}$ avaliação da influência da represa sobre os anofelinos, foi possível notar a complexidade estabelecida na interação entre esses mosquitos e o ambiente. A dinâmica adaptativa do gênero Anopheles foi induzida já nas primeiras mudanças, determinadas pelo desmatamento relativo à colonização da região. Este estado encontrado no nível da cota $247 \mathrm{~m}$, representado pelo leito do rio Paraná, induziu nos anos subseqüentes uma estabilidade na composição das espécies Culicidae, avaliada pelos resultados obtidos nas localidades A e B, sob influência de fatores atuantes antes das intervenções do empreendimento da hidrelétrica.

Nesse período, a população de An darlingi era composta de baixa freqüência de adulto e rara sob formas imaturas. Consolim e Galvão ${ }^{14}$ tiveram resultados similares no Rio Paraná, mais ao Sul, uma vez que encontrar An darlingi era um fato raro. Guimarães e cols ${ }^{15}$, ao pesquisarem em Guairá, $\mathrm{PR}$, nas proximidades do reservatório de Itaipu, estranharam não capturar An darlingi.

Os fatores ambientais no período pré-inundação correspondiam a duas localidades dominadas por lagoas e córregos que desaguavam no Rio Paraná. O solo era intensamente utilizado pela agricultura familiar e havia poucas manchas de vegetação vertical. No período chuvoso, formavam-se pequenas coleções líquidas nas depressões do solo e espécimes imaturos de An darlingi quase sempre estavam ausentes nesses habitats.

$\mathrm{Na}$ primeira inundação, que correspondeu à cota $253 \mathrm{~m}$, na localidade $\mathbf{B}$, emergiram formas imaturas e adultas de An darlingi. $\mathrm{Na}$ hidrelétrica Itaipu, a dificuldade anterior e a facilidade posterior de encontrar a espécie foram semelhantes ${ }^{15-17}$. Tais observações mostram que o aumento da densidade da espécie tem também relação com as chuvas e, portanto, no presente estudo da represa Porto Primavera, a adaptação de An darlingi deve, igualmente, depender de outros fatores, além dos determinantes diretamente ligados à formação do lago artificial ${ }^{14}$. Klein e Lima $^{18}$, estudando os mosquitos em Rondônia, relataram que a maior densidade ocorreu no final do período chuvoso, quando a água permanecia nas depressões naturais do solo, formando lagoas e pântanos temporários. Reconheceram, então, que a alta densidade está relacionada à abundância de alagados naturais e quantidade de vegetação aquática.

Na sequência de mudanças, observa-se uma queda rápida na densidade de An darlingi, porém; houve o recrudescimento de
An albitarsis s.l. e An triannulatus. Essa alteração revela a possível dificuldade da primeira espécie em ocupar os novos habitats do reservatório em processo de sucessão ecológica. Esses registros indicam haver mudanças desfavoráveis ao estabelecimento de An darlingi, sendo indicativo de retorno às densidades reduzidas detectadas antes da fase de alagamento do rio.

Para outras espécies, como An albitarsis s.l. e An triannulatus, o impacto ambiental foi respondido de forma diferente, pois ambos, embora mostraram oscilações em suas densidades, sinalizam para uma persistência destacada na área. $\mathrm{Na}$ localidade $\mathbf{C}$, essa característica foi mais nítida, coincidindo o mesmo efeito com outras pesquisas que envolveram o Rio Paraná e Lago Itaipu ${ }^{15,16}$. A espécie An rondoni, considerada o anofelino das enchentes ${ }^{19}$, apresentou aspecto coincidente apenas com a inundação da várzea da localidade $\mathbf{C}$. $\mathrm{O}$ mesmo fato não foi verificado nas demais localidades estudadas.

Nesse cenário, as duas inundações sucessivas devolveram à região a densidade anterior de An darlingi, pois apenas na cota $253 \mathrm{~m}$, na localidade $\mathbf{B}$, sua densidade foi elevada. Dentre os vários fatores ressalta-se que as terras altas do lado do Estado de São Paulo contribuíram para reduzir a chance de recrudescimento de An darlingi. Logo, a sobrevivência da espécie voltou a depender de criadouros em locais mais distantes para a realização das oviposições.

O índice de diversidade de Brillouin não apresentou divergência entre as localidades A e B, significando situações ecológicas semelhantes entre si $^{7}$. Com isso, An triannulatus e An albitarsis s.l. estavam mais representadas. No entanto, nas sucessivas inundações, a diversidade se alterou, mas foi na cota $253 \mathrm{~m}$, na localidade $\mathbf{B}$, que An darlingi tornou-se a espécie mais abundante, não atribuível à represa mas aos agrupamentos de formas imaturas, oriundas de diversos habitats disseminados pela área de estudo. Para a cota $257 \mathrm{~m}$, a diversidade elevou-se pelo resultado da localidade $\mathbf{C}$, ao contrário do que ocorreu com a $\mathbf{B}$, sendo que a representatividade específica foi para An darlingi, An triannulatus e An albitarsis s.l. A inundação na localidade $\mathrm{C}$, reproduziu o transbordamento do rio Paraná e a estabilização do lago nesse patamar eliminou esse este fenômeno, sinalizando a redução da densidade de An darlingi. Assim, os resultados aqui obtidos são concordantes com outras observações que explicam alterações na diversidade em razão das limitações dos habitats existentes ${ }^{20,21}$.

No período estudado, diante da turbulência sequencial das alterações do nível das águas, conclui-se que a represa Porto Primavera apresentou impacto adverso ao esperado, pois o aumento preliminar da densidade de An darlingi nas localidades $\mathbf{B}$ e $\mathbf{C}$ foi temporário. Consequentemente, o risco epidemiológico para recrudescimento da transmissão de plasmódios da malária humana não se confirmou, permanecendo a tendência inalterada ao entorno do açude, apesar da pressão de introdução de plasmódio manter-se pelos mais de oito mil casos importados registrados no estado durante o período de 1990 a $2006^{6}$. Assim sendo, alerta-se sobre a importação de plasmódio na área, ${ }^{1}$ ao se considerar que vetores secundários possam favorecer a passagem de infecção para primatas não humanos, elevando-se o risco de infecções humanas futuras provenientes desses primatas.

\section{AGRADECIMENTOS}

Ao Analista de Sistemas Fernão Dias de Lima pelo apoio estatístico. 


\section{CONFLITO DE INTERESSE}

Os autores declaram não haver nenhum tipo de conflito de interesse no desenvolvimento do estudo.

\section{SUPORTE FINANCEIRO}

Fundação de Amparo à Pesquisa do Estado de São Paulo (FAPESP), Brasil (96/10014-1; 99/11377-9). Logística de campo: Companhia Energética de São Paulo (CESP).

\section{REFERÊNCIAS}

1. Gomes AC, Paula MB, Duarte AMRC, Lima MA, Malafonte RS, Mucci LF et al Epidemiological and ecological aspects related to malaria in the area of influence of the lake at Porto Primavera dam, in western São Paulo State, Brazil. Rev Inst Med Trop Sao Paulo 2008; 50: 287-295.

2. Chaves KM, Zumpano JF, Resende MC, Pimenta FG, Rocha MOC. Malária em Minas Gerais, Brasil, no Período 1980-1992. Cad Saúde Pública 1995; 11: 621-623.

3. Limongi JE, Chaves KM, Paula MBC, Costa FC, Silva AA, Lopes IS, et al. Malaria outbreaks in a non-endemic area of Brazil, 2005. Rev Soc Bras Med Trop 2008; 41: 232-237.

4. Vittor AY, Gilman RH, Tielsch J, Glass G, Shields T, Lozano WS, et al. The effect of deforestation on the human-biting rate of Anopheles darlingi, the primary vector of falciparum malaria in the Peruvian Amazon. Am J Trop Med Hyg 2006; 74: 3-11.

5. Wanderley DMV, Andrade JCR, Alves MJCP, Alves MCGP, Mattos MR, Gurgel SM, et al. Malária no Estado de São Paulo: Avaliação de aspectos da vigilância epidemiológica Cad Saude Publica 1989; 5: 296-304.

6. Wanderley DMV, Ciaravolo RMC, Barbosa GL, Spínola R, Leite RM. Malária no Estado de São Paulo: Aspectos da Vigilância Epidemiológica. BEPA Bol Epidemiol Paul 2006; 3: 2-7.

7. Johnson MF, Gómez A, Pinedo-Vasquez M. Land use and mosquito diversity in the Peruvian Amazon. J Med Entomol 2008; 45: 1023-1030.

8. Tadei WP, Thatcher BD. Malaria vectors in the Brazilian Amazon: Anopheles of the subgenus Nyssorhynchus. Rev Inst Med Trop Sao Paulo 2000; 42: 87-94.

9. Póvoa MM, Conn JE, Schilichting CD, Amaral JCOF, Segura MNO, Silva ANM et al. Malaria vectors, epidemiology, and the re-emergence of Anopheles darlingi in Belém, Pará, Brazil. J Med Entomol 2003; 40: 379-386.

10. Loiola CCP, Mangabeira da Silva CJ, Tauil PL. Controle da malária no Brasil: 1965 a 2001. Rev Panam Salud Publica 2002; 11: 235-244.

11. Paula MB, Gomes AC. Culicidae (Diptera) em área sob influência de construção de represa no Estado de São Paulo, Brasil. Rev Saude Publica 2007; 41: 284 289

12. Gomes AC, Natal D, Paula M.B, Urbinatti PR, Mucci LF, Bitencourt MD. Riqueza e abundância de Culicidae (Diptera) em área impactada, Mato Grosso do Sul, Brasil. Rev Saude Publica 2007; 41: 284-289.

13. Magurran AE. Ecological diversity and its measurement. Cambridge: University Press; 1988.

14. Consolim J, Galvão JT. Sobre os anofelinos do rio Paraná. I Densidade e Regime do Rio. Arq Biol e Tecnol 1973; 16: 174-181.

15. Guimarães AE, Mello RP, Lopes, CM, Alencar J, Gentile C. Prevalência de anofelinos (Diptera:Culicidae) no crepúsculo vespertino em áreas da usina hidrelétrica de Itaipu, no município de Guairá, Estado do Paraná, Brasil. Mem Inst Oswaldo Cruz 1997; 92: 745-754.

16. Consolim J, Luz E, Pellegrini NJM, Torres PB. O Anopheles (Nyssorhynchus) darlingi Root, 1926 e a malária no lago de Itaipu, Estado do Paraná, Brasil: Uma revisão de dados. Arq Biol e Tecnol 1991; 34: 263-286.

17. Falavigna-Guilherme AL, Silva AM, Guilherme EV, Morais DL. Retrospective study of malaria prevalence and Anopheles genus in the area of influence of the binational Itaipu reservoir. Rev Inst Med Trop Sao Paulo 2005; 47: 81-86.
18. Klein TA, Lima JBP. Seasonal distribution and biting patterns of anopheles mosquitoes in Costa Marques, Rondonia, Brazil. J Am Mosq Control Assoc 1990; 6: 700-707

19. Consolim J. Sobre os anofelinos do rio Paraná. III Comportamento do Anopheles rondoni. Arq Biol e Tecnol 1974; 17: 113-114.

20. Hutchings RSG, Sallum MAM, Ferreira RLM, Hutchings RW. Mosquitoes of the Jau National Park and their potential importance in Brazilian Amazonia. Med Vet Entomol 2005; 19: 428-441.

21. Leisnham PT, Slaney DP, Lester PJ, Weinstein P. Increased larval mosquito densities from modified landuses in the Kapiti Region, New Zealand: vegetation, water quality, and predators as associated environmental factors. EcoHealth $2005 ; 2: 1-10$. 